\title{
Selective Trapping of Bacteria with Molecularly Imprinting Film under External Field
}

\author{
Shiho Tokonami ${ }^{1,2^{\star}}$,Emi Shimizu ${ }^{1,2}$, Mamoru Tamura ${ }^{2,3}$, and Takuya lida ${ }^{2,3}$ \\ ${ }^{1}$ Department of Applied Chemistry, Graduate School of Engineering, Osaka Prefecture University, \\ Sakai, Osaka 599-8570, Japan \\ ${ }^{2}$ Research Institute for Light-induced Acceleration System (RILACS), Sakai, Osaka 599-8570, Japan \\ ${ }^{3}$ Department of Physical Science, Graduate School of Science, Osaka Prefecture University, Sakai, \\ Osaka 599-8570, Japan \\ *tokonami@chem.osakafu-u.ac.jp
}

\begin{abstract}
:
The mechanism of selective trapping of macroscopic bacteria by the cavities on the surface of the bacterial imprinted film from was unsolved. Here, we discuss the physicochemical mechanism of rapid specific identification of bacteria using an alternating electric field and a quartz crystal microbalance. We have experimentally revealed that the selectivity strongly depends on the specific binding of surface chemical structure (SCS) and molecular recognition site (MRS) under the various conditions of SCS on bacteria damaged by UV light, heat and antibacterial drugs. We also performed the theoretical analysis based on the "self-consistent electromagnetic theory taking into account the MRS, and well explained the experimentally observed selectivity from the nanoscale physicochemical viewpoint.
\end{abstract}

Key words: Bacteria, Imprinted Film, Dielectrophoresis, Quartz Crystal Microbalance, Simulation

\section{Introduction}

The detection of nano-biomaterials and microbes are the crucial subject in the food safety and medical diagnosis, where the molecularly-functionalized nanospace and microspace are promising strategy. Paying attention to biosensor for bacteria, there are various types of passive and active manners available for specific detections of microbes [1]. For example, a structure of the target molecule can be transcribed into a surface of polymer film with molecular imprinting method, and a development of efficient molecular sensors has been reported [2]. This technique enables to create "bacterial imprinted film (BIF)" with a surface structure of target bacteria. It has been demonstrated that only target bacteria are specifically captured by BIF with the help of external alternating electric field, i.e., dielectrophoresis (DEP) even in a mixture liquid of various kinds of bacteria including the target ones and different ones with a similar shape. On the other hand, the physicochemical mechanism of selective trapping of bacteria is important but remains as an unresolved problem [3].
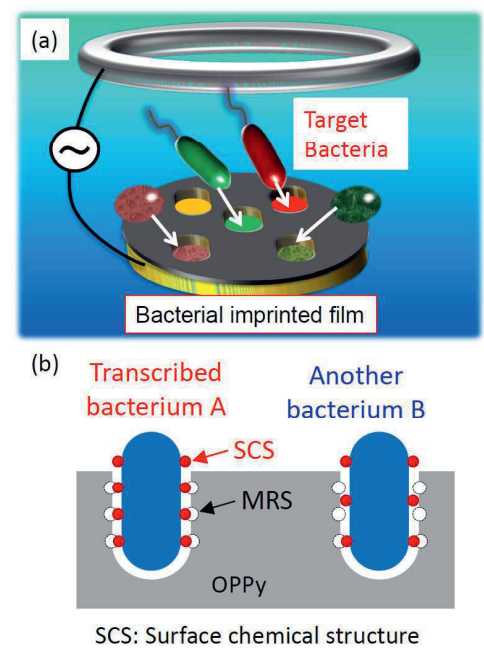

MRS: Molecular recognition site

Fig. 1. Schematic illustrations for (a) experiment of external-filed induced selective trapping of BIF and (b) Model for the theoretical analysis of complementary bacteria $A$ and mismatched bacteria $B$ for BIF of the cavity of bacteria A in OPPy.

Therefore, we tried to clarify the mechanism of such a selectivity by changing the surface condition of target bacteria with UV light, thermal treatment, and antibacterial drug, and 
theoretical analysis under the DEP taking into account the molecular recognition process.
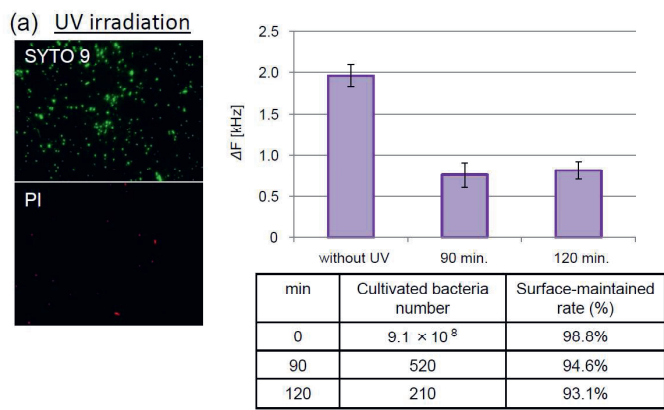

(b) $\{$ l $\} K_{\text {rec }}=2 \mu N / m$

$\{\mathrm{II}\} \mathrm{K}_{\mathrm{rec}}=3 \mu \mathrm{N} / \mathrm{m}$

$\{I I I\} \mathrm{K}_{\mathrm{rec}}=4 \mu \mathrm{N} / \mathrm{m}$
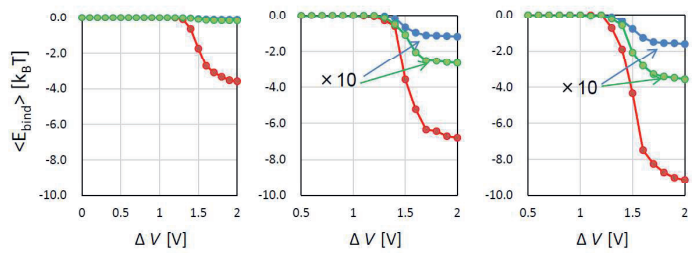

Complementary bacteria

- Mismatched bacteria <i>

Fig. 2. (a) Specific detection of outage bacteria (E. coli 0157:H7) damaged by ultraviolet (UV) light irradiation. Cultivated bacteria number and Surfacemaintained rate are summarized in the table. (b) Theoretical results for 3 types bacteria with different SCS.

\section{Methods}

We have produced a BIF with cavities imprinting the information of $\mathrm{E}$. coli group by electro-polymerization of over-oxidized polypyrrole (OPPy), and confirmed the selective trapping into the produced BIF via frequency change of quartz crystal microbalance (QCM) by guiding target bacteria toward the BIF on the electrode with DEP (Fig. 1 (a)). In addition, we develop theoretical method for the stochastic behavior of bacteria under DEP and the interaction at MRS, i.e. "Molecular recognition Metropolis method (MRMM)". For the calculation with MRMM, it is assumed that the surface chemical structures (SCS) of a bacterium are transcribed into each cavity of BIF and a complementary bacterium can be specifically recognized by the molecular recognition site (MRS) given via imprinting process (Fig. 1 (b)).

\section{Results and Discussion}

In order to confirm the selective trapping of living bacteria, a BIF was produced using bacteria (E. coli O157:H7) without surface damage. Using this BIF under alternating electric field, the non-damaged bacteria without
UV irradiation can be detected via the frequency change of $\mathrm{QCM}$ on the order of 2 kHz (Fig.2(a)). However, after UV irradiation for over $90 \mathrm{~min}$, the frequency change was significantly decreased even in the case of using complementary bacteria. Also, the cultivated number of bacteria number did not increase by damaging the bacteria with UVirradiation. The surface-maintained rate was estimated from the bacterial number of stained with SYTO 9 and propidium iodide (PI) exhibiting red fluorescence. Only when the cell membrane was broken, PI can enter the inside of cells. The fluorescent image shows that the outer membrane was not damaged. On the other hand, thermal treatment shows large amount of bacteria shows red fluorescence of $\mathrm{PI}$ and there was no frequency change of QCM. Moreover, when antibacterial drug penetrate into the bacterium without damage in the surface, the frequency change was maintained. It was considered that such a selectivity can be attributed to the SCS of bacteria, where the surface sugar chain was broken by UV irradiation. In Fig. 2(b), we have performed the simulations with MRMM and successfully explains the selective trapping of complementary bacterium under DEP. This means that the spatial configuration and binding strength of MRS and SCS is important for the selectivity. Based on such a principle, we also confirmed the highly selective detection of various bacteria with mixed-type BIF.

The discussion here was based on the fundamental physicochemical mechanism, whereas our clarified mechanism will pave the way to a construction of guiding principle to design a highly sensitive and rapid bacterial sensor with BIF.

\section{References}

[1] S. Tokonami, Takuya lida, "Review: Novel Sensing Strategies for Bacterial Detection Based on Active and Passive Methods Driven by External Field", Analytica Chimica Acta, Vol.988, 1-16. (2017). DOI:10.1016/j.aca.2017.07.034

[2] S. Tokonami, Y. Nakadoi, M. Takahashi, M. Ikemizu, T. Kadoma, K. Saimatsu, L. Q. Dung, H. Shiigi, and T. Nagaoka, "Label-free and selective bacteria detection using a film with transferred bacterial configuration", Analytical Chemistry 85, 4925 (2013). DOI: $10.1021 /$ ac3034618

[3] S. Tokonami, E. Shimizu, M. Tamura, and T. lida, "Mechanism in External Field-mediated Trapping of Bacteria Sensitive to Nanoscale Surface Chemical Structure", Scientific Reports 7, 16651 (2017). DOI:10.1038/s41598-017-15086-1 